\title{
EDITORIAL
}

\section{Tracking social challenges for pharmaceutical education in Brazil during the COVID-19 pandemic}

\author{
Silvana Nair Leite, Luciano Soares \\ Federal University of Santa Catarina, Brazil
}

\author{
Correspondence \\ Silvana Nair Leite \\ Campus Trindade \\ Department of Pharmaceutical \\ Sciences \\ Federal Univerty of Santa Catarina \\ CEP 88040010 \\ Florianópolis \\ Brazil \\ silvana.nair@hotmail.com
}

\begin{abstract}
Multiple successful experiences in overcoming the challenges of offering urgent distance learning during the COVID-19 pandemic have been reported in international literature. However, the use of distance learning in pharmaceutical education all over the world is both recent and limited to a supporting role in face-to-face classes. The migration of all pedagogical activities to the distance learning mode in Brazil raises particular worries. It is necessary to analyse the scenarios of the pandemic and of higher education in Brazil, as well as the scenario of pharmaceutical education, to understand the concerns of professors and students. This understanding is also key to glimpse ways of surmounting the numerous difficulties presented in these scenarios.
\end{abstract}

On 3rd February 2020, the Brazilian government declared a national public health emergency due to human infection by the new Coronavirus (COVID-19). Due to the public health situation, face-to-face classes in Brazil were suspended in March 2020. The academic year had just begun at that time. The Ministry of Education issued a decree, authorising the substitution of regular classes for distance learning/online teaching in higher education. The decree also established that professional practice internships and practical classes in specialised labs would be substituted for the use of digital educational resources until December of 2020. These replacement activities have to follow the National Curricular Guidelines (Decree no. 544/2020) (Government of Brazil, 2020).

Multiple successful experiences in overcoming the challenges of offering urgent distance learning during the pandemic have been reported in the international literature (Dedelilia et al., 2020). However, the use of distance learning in pharmaceutical education all over the world is both recent and limited to a supporting role in face-to-face classes (Lorenzoni et al., 2019). The migration of all pedagogical activities to the distance learning mode in Brazil raises particular worries. It is necessary to analyse the scenarios of the pandemic and of higher education in Brazil, as well as the scenario of pharmaceutical education, to understand the concerns of professors and students. This understanding is also key to getting an insight into ways of surmounting the numerous difficulties presented in these scenarios.

\section{The scenario}

On 10th September 2020, Brazil reached the mark of almost 4.2 million cases of COVID-19 and approximately 130,000 deaths. The absence of robust public policies to face the pandemic in the country has already been making national and international headlines. The economy has had a significant recession since March. Because of the COVID-19 pandemic, 10.9 million people are unemployed 
and 30.2 million employed people have had their income reduced. This scenario deepens the country's rampant inequality even further. It also affects the abilities of students to engage with their educational activities at home, since very often, they face a combination of sickness, death, or financial difficulties.

In 2018, the Brazilian Institute of Geography and Statistics reported that only $41.7 \%$ of Brazilian households in urban areas had a personal computer and only $12.5 \%$ had a computer tablet. One out of five Brazilians did not have access to the Internet at home.

In Brazil, more than 600 Higher Education Institutions (HEls) offer Bachelor of Pharmacy programmes, most of them $(85 \%)$ are privately funded institutions, and some are very small institutions. They are evaluated by the Ministry of Education in a triennial process. The evaluation results in 2016 (regarding student performance, faculty, infrastructure, and didactic and pedagogical organisation) show that $6.4 \%$ of all pharmacy schools received an unsatisfactory score. The majority of these were private institutions. The mean score of the schools is three (only sufficient). The majority (60.0\%) of the pharmacy schools that achieved the maximum score were publicly funded (Lopes et al., 2019). Even though they receive three out of four enrolled students, the private institutions employ only a little more than half of the professors. Around 68\% of the professors who hold a Ph.D. degree are employed in public institutions. The resources for research and substantive postgraduate programmes in pharmacy are concentrated only in public universities. Hence, most of the pharmacy schools perform poorly in research, post graduation, and innovation.

Since 2001, the higher education courses offered in the traditional mode in Brazil are authorised to have up to $20 \%$ of the total academic load through distance learning activities. In 2018, the limit was extended to $40 \%$ for every higher education institution, with the only exception being medical degrees. From that moment on, there has been a strong movement from professional institutions, professors, and students, requiring more strict regulation for distance learning, with clear limits established for its use in the health field.

The challenges for the Brazilian pharmaceutical education in these critical times of the pandemic reflect the characteristics of these scenarios and relate to four key questions.

\section{Academic capacity}

The infrastructure of many institutions, including those with the best assessment, is insufficient. There is a lack of hardware and communication software, as well as of specific pedagogical tools for teaching, in the field of pharmacy. The institutions have been investing in the impromptu acquisition of proprietary tools to be able to meet the extreme rise of their demand.

The faculties in these institutions must deal with the predicament of adapting their pedagogical praxis to the new reality. Their difficulties with this process are very often a result of the level of digital literacy, of a compounded traditional didactic culture, as well as of the institutional limits concerning infrastructure and the availability of appropriate instruments. Many professors are teaching online classes for the first time. These issues have forced institutions to organise emergency faculty training programmes.

\section{Students' digital access}

Students' access to the Internet and equipment (computers, smartphones, webcams) frequently features as an obstacle to distance learning in the results of national surveys. A lack or low quality of equipment, as well as Internet services with limited or unstable connections, are the reality of many students.

Multiple educational institutions have structured programmes to help students overcome such obstacles. These programmes provide Internet data plans for their students and support their purchasing of equipment. Some public universities are fostering large programmes of lending computers to the poorest students.

\section{Laboratory practice}

The pedagogical strategies with the greatest didactic sophistication in pharmacy courses in Brazil involve the practices that take place in specialised labs in the institutions, including active methods that support students' learning. The permission to substitute $100 \%$ of this kind of activity for remote alternatives incurs limitations in the learning of professional skills, thus, creating a gap in a crucial dimension for the development of the competencies expected from a pharmacist. Additionally, there are not a lot of specialised resources available in Brazil to create a remote experience that can reasonably substitute on-site activities. Many schools are simply recording the practical activities in demonstration videos and making them available to students, who take a passive role as the audience. Some universities chose not to offer the practical classes in 2020 and these course credits are only going to be offered when the regular activities return. 


\section{Internships/experiential learning}

The Brazilian Pharmaceutical Education Guidelines define that experiential learning must be offered from the beginning years of a course and must include internships in health service provision in the last years before graduation. A minimum of $20 \%$ of hours during the undergraduate programmes must be in internship activities. The universities are in charge of providing the conditions for supervising and evaluating these activities. Current guidelines emphasise that pharmacist training should comprise social health needs, comprehensive care, and teamwork, especially in the Brazilian Unified Health System (Leite et al., 2020).

The authorisation for internships to take place remotely, through digital tools, is an insufficient adaptation as it lacks the capability of promoting the necessary professional experience. The pharmaceutical professional practice in Brazil mostly happens on-site. The pandemic certainly sped up the changes in work processes towards digitalisation, however, the sociodemographic characteristics of the country show that on-site services will remain fundamental.

In contrast, while allowing universities to replace in-person internships, the government created the "Brasi Conta Comigo" programme, a national programme that finances the hiring of students (from the final years of the courses, including pharmacy) as interns, to work on the frontline of health services without academic supervision and with insufficient security protocols.

\section{The future}

Information and communication technologies and the strategies for distance learning constitute valuable resources to the evolution of pharmaceutical education in Brazil. However, the educational system needs to find strategies to prevail over the current social conditions. The lessons from these circumstances should motivate academia, professionals, and Brazilian society to reflect upon the investments in the pharmaceutical education, aiming at connecting the instruction and the profession to the scientific and technological advances which are already ubiquitously spread in the twenty-first century.

\section{References}

Dedelilia, A.D., Sotiropoulos, M.G., Hanrahan, J.G., Janga, D., Dedeilias, P., \& Sideris, M. (2020). Medical and Surgical Education Challenges and Innovations in the COVID-19 Era: A Systematic Review. In Vivo 34(3), 1603-1611. http://doi: 10.21873/invivo.11950
Government of Brazil. (2020). Ministério da Educação. Portaria № 544/2020. Available at: https://www.in.gov.br/en/web/dou/-/ portaria-n-544-de-16-de-junho-de-2020-261924872

Leite, S.N., Rover, M.R.M., Soares, L., \& Matheus, F.C. (2020). Losses and gains in experiential education in a university pharmacy in Brazil: Lessons from a pandemic. Pharmacy Education, 20(3), 39-40. https://doi.org/10.46542/pe.2020.202.3940

Lopes, N.M.S., Gondim, A.P.S., Soares, A.C.S., Santos, D.B., Sales Neto, M.R., \& Pinto, D.M. (2019). A quantitative analysis of the quality fo pharmacy education in Brazil. American Journal of Pharmaceutical Education, 83(3). https://doi.org/10.5688/ ajpe6543

Lorenzoni, A.A., Manzini, F., Soares, L., \& Leite, S.N. (2019). E-learning in Pharmacy Education: what do we know about it? Brazilian Journal of Pharmaceutical Sciences, 55. https://doi.org/ $\underline{10.1590 / \mathrm{s} 2175-97902019000118100}$ 